\title{
Railway fastener defects recognition algorithm based on computer vision *
}

\author{
Jiajia Liu \\ School of Mechanical Engineering \\ Southwest Jiaotong University \\ Chengdu, Sichuan \\ E-mail: liujia9437@126.com
}

\author{
Bailin Li \\ School of Mechanical Engineering \\ Southwest Jiaotong University \\ Chengdu, Sichuan \\ E-mail: blli62@263.net
}

\begin{abstract}
Railway fastener detection is an important task in railway maintenance to ensure safety. However, the earlier detection methods based on computer vision have good performance on missing fasteners, but they have weaker ability to recognize the partially worn ones. In this paper, we exploit the axis-symmetrical structure to generate the first and second symmetry sample of original testing fastener image, and integrate the first and second image for improved representation-based fastener recognition. The underlying advantages of the scheme are as follows: first, the symmetry image can somewhat overcome the difficulty that the lack of training and testing samples. Second, the symmetry image is helpful for representation-based fastener recognition and we can obtain an accurate judgment of the original testing image by integrate the corresponding judgments of two symmetry image. The experiment results show that our proposed method can achieve a rather high precision.
\end{abstract}

Keywords-Railway fastener detection; Symmetrical image; Computer vision; Harr-like feature;Improved sparse representation.

\section{INTRODUCTION}

Railroads conduct regular inspection of the track is a very critical task ensure the safety of train traffic. Railway fastener defect detection is an important part of the regular inspection, the causes of two common quality problems are partly worn and missing fastener, as shown in Figure.1, which would cause the train derailment and even threaten the safety of train operation. Recently, the researchers are interested in developing automatic inspection methods based on computer vision. For hook-shaped fasteners, Yang et al. [1] took advantages of direction field as the template of fastener. Similarly, Stellaet al. [2] employed wavelet transform and principal component analysis to preprocess railway images and used the neural classifier to recognition the missing fasteners. To achieve real-time performance, Ruvo et al. [3] applied the error back propagation algorithm to model the fasteners and automatic fastener detection. However, the main work of the researches above aim at searching for missing fasteners and they are difficult to detect the partly worn fastener. Recently, P.L. Mazzeo, et al. [4] and Rubinsztejn [5] successfully applied the Adaboost algorithm to the fastener detection. Specifically, Xia, et al. [6] departed the hookshaped fastener into four parts and each part was independently trained by Adaboost. Similarly, Li et al. [7] used image processing methods to detect the components of fastener. Other technologies that have been used for detecting fasteners include support vector machine (SVM) [8] and Gabor filters [9]. In summary, the majority of earlier methods

Identify applicable sponsor: National Natural Science Foundation of China (Grant No. 51305368), Science and technology support project of Sichuan province (Grant No.2012GZ0102 and No.2014GZ0005), Doctoral innovation foundation of southwest jiaotong university (Grant No.2014). take advantage of image feature and discriminative classifiers to classify the testing fastener samples, and they achieve a good performance in fastener defect identification, however, they have weaker ability to identify the partially worn fasteners. Up to now, fastener recognition is still confronted with a number of challenges such as:

1) Limited defective samples used for training classifier. Railway fastener defects are roughly divided into two categories: partially worn and missing fasteners. And the partially worn fasteners appear in an arbitrary manner, i.e., there is no uniform representation of the defective cases. It seems that more training samples are beneficial for fastener recognition; however, there are only a limited number of defective instances on the actual railway line.

2) Illumination inequality and image noise. Fastener images are captured by a camera installed under a moving track checking train in an open circumstance, the illumination in an image is ready to be changed because of natural light, and the image is contaminated seriously by random noise. Affected by these factors, the distinctiveness of fastener image feature and the effective of the fastener detection method are also restricted.

In order to address these challenges and obtain better recognition result in fastener classification and recognition, many scholars have made many efforts in improve the image feature and increase training samples. However, the special nature of the fastener is neglected, such as the symmetry of the structure in fastener recognition. This motivates us to exploit the symmetrical structure of the fastener and devise a representation based method to perform fastener recognition, and our method is designed for finding both the partially worn and the completely missing instances. This paper has the following main contributions: the idea of image symmetry being introduced into fastener recognition for the first time, it is a simple way to obtain more training and testing samples by the symmetrical operation. Moreover, we turn the detection of the original testing image into the detection of two symmetrical images and devise an improved representation based classification algorithm for fastener detection. We also provide many experiments and the experimental results show that the proposed method is very competitive.

The remainder of this paper is organized as follows. In Section 2, we provide the fastener detection method based on the symmetric fastener image. In Section 3, our methods are verified by extensive experiments using fastener databases. Section 4 offers our conclusion. 


\section{THE PROPOSED METHOD FOR FASTENER DETECTION}

We first generate two symmetrical fastener images from the original sample. Let $y_{i}$ and $z_{i}$ respectively stand for the first and second symmetrical sample generated from the original sample $t_{i}$. The left half columns of $y_{i}$ is set to the same as that of $t_{i}$ and the right half columns of $y_{i}$ is the mirror image of the left half columns of $y_{i}$. However, the right half columns of $Z_{i}$ is set to the same as that of $t_{i}$ and the left half columns of $Z_{i}$ is the mirror image of the right half columns of $Z_{i}$.
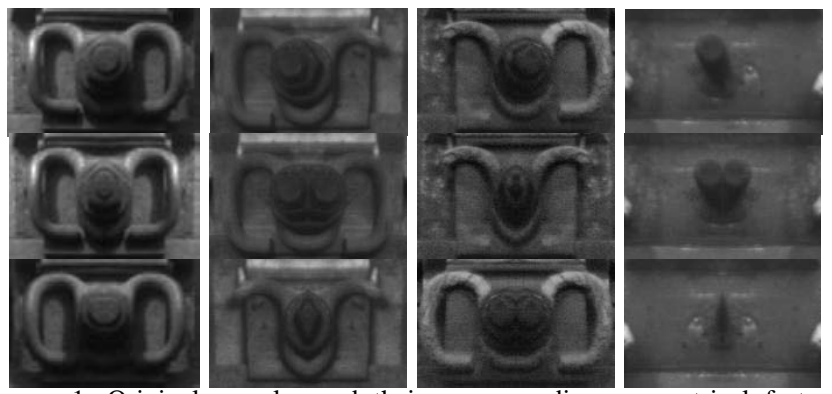

Figure. 1. Original samples and their corresponding symmetrical fastener samples. The first row shows the original samples; the second and third row respectively shows the first and second 'symmetrical fastener' samples.

Figure.1 shows some original samples and their corresponding symmetrical fastener samples. We can see that the symmetrical image has the following advantages:

First, though the 'symmetrical fastener' image is simply generated from the original fastener image, it also appears to be a natural fastener image and properly reflects some possible appearance of fastener image in image poses and illumination, especially the 'symmetrical fastener' image generated from the partially worn fastener image, which are not shown by the original samples. Moreover, we also find that the major difference between intact and defective fasteners lies in the four end regions of fastener, therefore, we regard the four local regions as the effective region of interest (ROI) and extract image feature for fastener recognition, it is not only ensure the discriminative of image feature, but also improve calculation efficiency of the propose detection method.

Second, the use of symmetrical image is helpful for the recognition of the partially worn fastener image. As the samples shown in the third column in Figure 1, the left branch

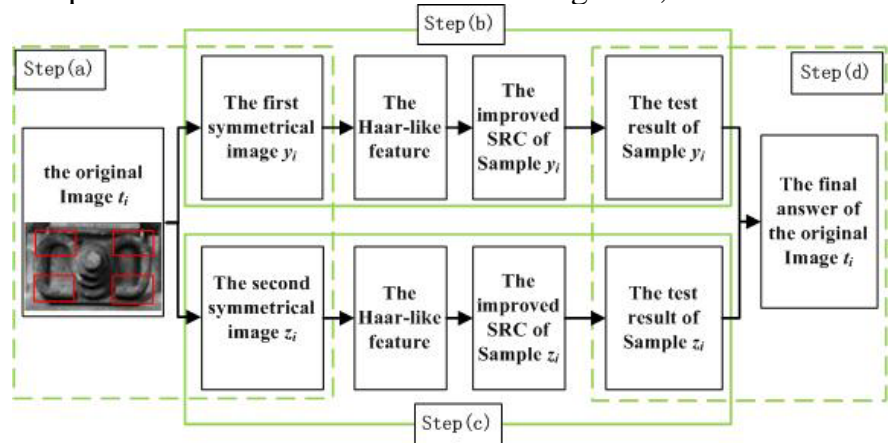

Figure. 2 the detection process of testing fastener. (a) Generate the first and second symmetrical samples. (b) The improved sparse representation of the first symmetrical fastener. (c) The improved sparse representation of the second symmetrical fastener. (d) Integrate the results obtained by the Step (b) and Step (c) to conduct fusion decision of original testing fastener. of the original testing sample is partially broken, and the appearance of its first symmetrical fastener looks more closely to the subject of partially broken fastener, On the other hand, its second symmetrical fastener looks same to intact fastener. Therefore, it is helpful the classifier to accurately classify the first symmetrical image or the second symmetrical image, then integrate the two decision results to make a final judgment of original testing fastener, it is able to reduce the side-effect of the error identification problem of the original fastener image.

These motivate us to exploit the symmetry of fastener to generate two new 'virtual samples' of test fastener and devise a representation based method to perform fastener recognition. The flow chart of our method as following:

As shown in Figure. 2, we Integrate the results obtained using the Step (b) and Step (c) to conduct fusion decision of original testing fastener, the decision rule as shown in Table 1. For example, if the first and the second symmetrical sample of the original testing fastener are identified as intact and partially worn fastener, respectively, then the ultimate status of original testing fastener is judged as partially worn fastener.

Table 1 the decision rule for the ultimate status of original testing fastener

\begin{tabular}{|c|c|c|}
\hline \multirow{2}{*}{ The status of $y_{i}$} & The status of $z_{i}$ & The ultimate status of $\boldsymbol{t}_{\boldsymbol{i}}$ \\
\hline \multirow{4}{*}{ intact } & intact & intact \\
\cline { 2 - 3 } & partially worn & partially worn \\
\cline { 2 - 3 } & missing & partially worn \\
\hline \multirow{3}{*}{ partially worn } & intact & partially worn \\
\cline { 2 - 3 } & partially worn & partially worn \\
\cline { 2 - 3 } & missing & partially worn \\
\hline \multirow{4}{*}{ missing } & intact & partially worn \\
\cline { 2 - 3 } & partially worn & partially worn \\
\cline { 2 - 3 } & missing & missing \\
\hline
\end{tabular}

A. Haar-like feature extraction of fastener image

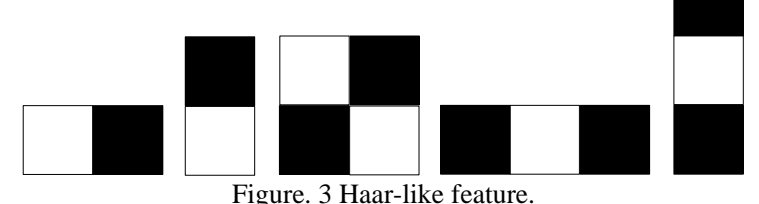

Figure. 3 Haar-like feature.

For a fastener image, the human eye can only distinguish the grayscale intensity difference between the image blocks such as the fastener and surrounding background region, in order to well describe the macro-texture feature, we adopt the improved Haar-like feature and utilize the integral Image to reduce the computation cost. Haar-like feature [10] is a simple but effective representation of local structures. To represent structures of multiple scales, we use five layouts as shown in Figure. 3, and link the Haar-like features of three different types with the size of 4-8 pixels into 45-dimension descriptor in our implementation. The multisize Haar-like feature is normalized according to the maximum values of each dimension. For example, the value of the first layout in Fig. 3 can be calculated using the following equation:

$$
\left|\sum_{j=0}^{H-1} \sum_{i=w}^{2 W-1} I(\mathrm{i}, \mathrm{j})-\sum_{j=0}^{H-1} \sum_{i=0}^{W-1} I(\mathrm{i}, \mathrm{j})\right| / \sum_{j=0}^{H-1} \sum_{i=0}^{W-1} 255
$$

Where $W$ and $H$ are the width and height of the subrectangle and $I(i, j)$ is the intensity value at the coordinate 
$(i, j)$. Other dimensions are normalized using the similar manner.

\section{B. The improved sparse representation algorithm}

In this section, we design an improved sparse representation algorithm for fastener image recognition, and it has a low computational cost relative to the conventional SRC algorithm [11, 12]. This method first uses all the training samples of every class to represent the test sample and exploits the representation ability so as to identify the $K$ nearest neighbors of the test sample, then represents the test sample as a linear combination of the 'nearest $\mathrm{K}$ neighbors' and uses the representation result to perform classification. For simplicity of presentation, we describe the algorithm only on the first symmetrical fastener, and the same algorithm on the second 'symmetrical fastener' is the same except replace 'the first symmetrical fastener image' with 'the second symmetrical fastener image'. The algorithm is described as:

$$
\tilde{y}=f_{i, 1} \tilde{x}_{1}+f_{i, 2} \tilde{x}_{2}+\ldots+f_{i, N} \tilde{x}_{N}
$$

Where $\mathrm{y}$ is the test sample and $f_{i, j}$ is the coefficient. Let $X=\left[\tilde{x}_{i, 1} \ldots \tilde{X}_{i, j} \ldots \tilde{X}_{i, N}\right]$ and $F=\left[f_{i, 1} \ldots f_{i, j} \ldots f_{i, N}\right]^{T}$. We rewrite (1) as following:

$$
\tilde{y}=X F
$$

$F$ is calculated using $\bar{F}=\left(X^{T} X+\mu I\right)^{-1} X^{T} \tilde{y}$. $\bar{F}=\left[\bar{f}_{i, 1}, \ldots, \bar{f}_{i, j}, \ldots, \bar{f}_{i, N}^{-}\right]^{T} \cdot \mu$ is a small positive constant and $I$ is the identity matrix. Equation (1) implies that the effect on representing the test sample of the $i$-th sample can be evaluated using

$$
d_{i}=\left\|\tilde{y}-\sum_{j=1}^{N} \tilde{f}_{i, j} \tilde{x}_{i, j}\right\|^{2}
$$

Equation (1) shows that the $j$-th training sample makes its own contribution $f_{i, j} x_{i, j}$ in representing the test sample y, and the contribution can be also assessed by the residual of the test sample with respect to the $j$-th training sample, i.e., $d_{i, j}=\left\|\tilde{y}-\tilde{f}_{i, j} \tilde{x}_{i, j}\right\|^{2}$. In other words, the effect on representing the test sample of the $j$-th training sample is somewhat similar to the distance between the $j$-th training sample and the test sample, and a small $d_{i, j}$ means that the $j$-th training sample has a great contribution in representing the test sample. We exploit $d_{i, j}$ to identify the $\mathrm{K}$ training samples in the $i$-th subject that have the $\mathrm{K}$ greatest contributions, then uses a linear combination of the first $K$ candidate training samples to precise represent the test sample. If the first $K$ candidate training samples are denoted by $x_{i, 1}, x_{i, 2}, \ldots, X_{i, K}$, respectively, then the algorithm is rewrite as:

$$
\tilde{y}=f_{i, 1} \tilde{x}_{i, 1}^{\prime}+f_{i, 2} \tilde{x}_{i, 2}^{\prime}+\ldots+f_{i, k} \tilde{x}_{i, \mathrm{~K}}^{\prime}
$$

Where $f_{i, k}$ is the coefficient. The solution process of (4) is similar as above mentioned. Suppose that $\tilde{x}_{i, 1}^{\prime}, \ldots, \tilde{X}_{i, K}^{\prime}$ and $f_{i, 1}, \ldots, f_{i, \mathrm{~K}}$ respectively stand for the training samples and the coefficients. The ultimate effect on representing the test sample of the $i$-th subject of sample can be evaluated using:

$$
\operatorname{dev}_{i}=\left\|\tilde{y}-\sum_{j=1}^{K} \tilde{f}_{i, j} \tilde{x}_{i, j}^{\prime}\right\|^{2}
$$

If $\mathrm{r}=\arg \min d e v_{i}$, then test sample $\mathrm{y}$ is ultimately assigned to the $r$-th class. In this paper, both $\mu$ and $\gamma$ were set to 0.015 in the experiments.

\section{RESULTS AND DISCUSSION}

In this section we evaluated the effectiveness of our methods by performing experiments on railway fasteners database. The database consists of 1800 gray images from three subjects such as intact fastener, partially worn fastener and missing fastener. Among them the training samples have 300 samples including original images and the symmetrical images, each class of training sample providing 100 images, the remaining 1500 original images used as testing sample and each class providing 500 images. Precision is a widely used criterion in pattern recognition [13] and it is defined as:

$$
\text { Precision }=\frac{T_{p}}{N}
$$

Where $T_{p}$ denotes the number of correct recognition, $N$ is the total number of the test samples in every subject.

\section{A. Experiment of fastener detection method}

We first verify the effectiveness of image symmetrical operation method by the experiments. We randomly select 100 testing samples that the left branch of fasteners are partially broken, and generate their first and second symmetrical sample via image symmetry operation, then calculate the interclass Chi-square distances between every 100 images of each class and intact fastener images in training sets. As the test result shown in Figure 4, the first symmetrical distance is much always bigger than the original distance and the second symmetrical distance is always much smaller than the original distance. That means that it is very helpful for a "distance" based classifier to accurately classify the first and second symmetrical image according to the distance between them and intact fastener, respectively, and then integrate the two judgments to make a final decision of original testing fastener.

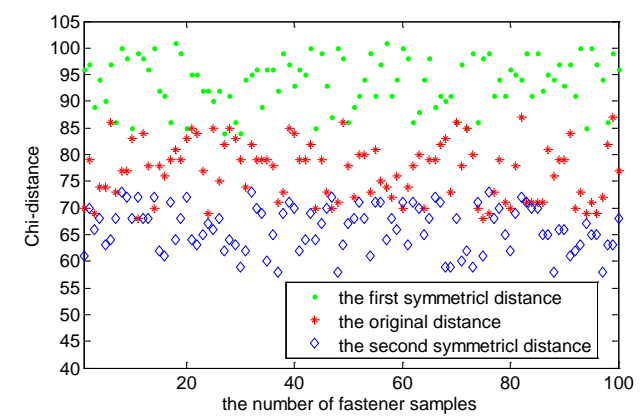

Figure 4 the Comparison between the original and symmetrical distance

To better illustrate the advantages of the image symmetry operation method, we also display the classification results of different methods, it should be noted that the fasteners of the same type are counted separately. We use SRC as the classifier and integrate the image symmetrical operation 
Table 2. The recognition precision (\%) and computation time of different methods on the fastener database.

\begin{tabular}{|l|c|c|c|c|c|c|c|c|c|c|c|c|}
\hline & \multicolumn{3}{|c|}{ intact fastener } & \multicolumn{3}{c|}{ partially worn fastener } & \multicolumn{3}{c|}{ missing fastener } \\
\hline SRC & \multicolumn{3}{|c|}{93.5} & \multicolumn{3}{c|}{92.8} & \multicolumn{3}{c|}{91.8} \\
\hline CPU time & \multicolumn{3}{|c|}{1.78} & \multicolumn{3}{c|}{1.72} & \multicolumn{3}{c|}{1.77} \\
\hline & $\mathbf{K = 1 0}$ & $\mathbf{K = 2 0}$ & $\mathbf{K = 5 0}$ & $\mathbf{K = 1 0 0}$ & $\mathbf{K = 1 0}$ & $\mathbf{K = 2 0}$ & $\mathbf{K = 5 0}$ & $\mathbf{K = 1 0 0}$ & $\mathbf{K = 1 0}$ & $\mathbf{K = 2 0}$ & $\mathbf{K = 5 0}$ & $\mathbf{K = 1 0 0}$ \\
\hline S-SRC & 93.3 & 96.3 & 95.8 & 94.5 & 94.3 & 98.5 & 96.5 & 94.6 & 94.8 & 96.8 & 94.8 & 93.2 \\
\hline CPU time & 0.37 & 0.49 & 0.71 & 0.89 & 0.36 & 0.50 & 0.73 & 0.86 & 0.35 & 0.48 & 0.72 & 0.87 \\
\hline
\end{tabular}

scheme and SRC is referred to as S-SRC. As the experiments shown in Table 2, we can see that the symmetrical operation of fastener image can greatly improve SRC method and obtain a higher recognition precision for all types of fasteners, especially for partially worn fastener, for example of that, the Precision of SRC method is $92.8 \%$, however, the corresponding precision of $\mathrm{S}-\mathrm{SRC}$ is $98.5 \%$ when the parameter $\mathrm{K}=100$.

Moreover, we see that when the number of $\mathrm{K}$ is smaller, our method almost always obtains a higher precision in contrast to traditional SRC, this clearly shows that it is really beneficial for the algorithm exploits the ' $K$ nearest neighbors' of the testing sample for the fine detection of it. Though we add a procedure to find the $\mathrm{k}$ candidate sample of the testing sample, but the additional procedure has quite low computational complexity, and it can significantly reduce the scale of the 12-minimization problem solved in performing SRC. Therefore, the total computational cost of our method decreases, which is verified in the experimental results as shown in Table 2.

\section{B. Performance comparison with different methods}

As can be seen from Table 3, the average recognition precision of adaboost [5], SVM [8], STM [14] and our method are $91.7 \%, 90.8 \%, 91.3 \%$ and $97.2 \%$, respectively, the proposed method outperforms the other methods on the same fastener databases. This indicates that the symmetry operation of fastener and sparsity are helpful in improving the classification accuracy for fastener recognition, and our method can avoid such misclassifications and improve the recognition precision of the partially worn fasteners to a great extent, thus improve the whole precision of fastener defect detection.

Table 3 the recognition precision (\%) of different methods

\begin{tabular}{|l|c|c|c|c|}
\hline \multirow{2}{*}{ Method } & \multicolumn{3}{|c|}{ Precision } & \multirow{2}{*}{$\begin{array}{c}\text { Average } \\
\text { precision }\end{array}$} \\
\cline { 2 - 4 } & $\begin{array}{c}\text { intact } \\
\text { fastener }\end{array}$ & $\begin{array}{c}\text { partially } \\
\text { worn } \\
\text { fastener }\end{array}$ & $\begin{array}{c}\text { missing } \\
\text { fastener }\end{array}$ & \\
\hline adaboost & 94.8 & 87.3 & 92.8 & 91.7 \\
\hline SVM & 93.5 & 86.5 & 92.5 & 90.8 \\
\hline STM & 92.3 & 87.8 & 93.8 & 91.3 \\
\hline $\begin{array}{l}\text { Our } \\
\text { method }\end{array}$ & 96.3 & 98.5 & 96.8 & 97.2 \\
\hline
\end{tabular}

\section{CONCLUSIONS}

We propose a novel railway inspection method in this paper, which is able to detect all types of damaged fastener. Theoretical analysis and the experiments are all shows that the symmetrical image and improved sparse representation are help for enhance the detection ability of fastener detection method, It can greatly eliminate the side-effect on the classification of the test sample and greatly improve the accuracy of the representation-based classification methods. We can conclude that our algorithm perform well in the detection of similar target which with a symmetrical structure.

\section{REFERENCES}

[1] J. Yang, W. Tao, M. Liu, Y. Zhang, H. Zhang, and H. Zhao. “An efficient direction field-based method for the detection of fasteners on high-speed railways”. Sensors. 2011. 8: 7364-7381.

[2] E. Stella, P. Mazzeo, M. Nitti, G. Cicirelli, A. Distante, and T. D’Orazio. "Visual recognition of missing fastening elements for railroad maintenance,". IEEE 5th International Conference on Intelligent Transportation Systems. Singapore, 2002. pp. 94-99.

[3] P. De Ruvo, A. Distante, E. Stella, and F. Marino. “A GPU-based vision system for real time detection of fastening elements in railway inspection,”. IEEE 16th International Conference on Image Processing. Cairo: Egypt, 2009. pp.2333-2336.

[4] P.L. Mazzeo, M. Nitti, E. Stella. Visual recognition of fastening bolts for railroad maintenance. Pattern Recognition Letter. 2004. 6. 669677.

[5] Y. Rubinsztejn. Automatic detection of objects of interest from rail track images. M.S. thesis, School Comput. Sci., Univ. Manchester, Manchester, U.K., 2011.

[6] Y. Xia, F. Xie, Z. Jiang. Broken railway fastener detection based on adaboost algorithm. International Conference on Optoelectronics and Image Processing. Hainan:China, pp.2010. 313-316.

[7] Y. Li, C. Otto, N. Haas, Y. Fujiki, and S. Pankanti. Component-based track inspection using machine-vision technology. Proceedings of the 1st ACM International Conference on Multimedia Retrieval. Vancouver, Canada, 2011. Article no. 60.

[8] P.L. Mazzeo, N. Ancona, E. Stella, and A. Distante. "Visual recognition of hexagonal headed bolts by comparing ICA to wavelets,” IEEE International Symposium on Intelligent Control. Houston:USA, 2003. pp.636-641.

[9] C. Mandriota, M. Nitti, N. Ancona, E. Stella, and A. Distante. Filter based feature selection for rail defect Detection. Machine Vision and Application. 2004. 4: 179-185.

[10] M. E. B. Sudderth, A. Torralba, W. T. Freeman, and A. S. Willsky, “Describing visual scenes using transformed objects and parts,” Int. J. Comput. Vis., 2008,77, pp. 291-330.

[11] J. Wright, Y. Ma, J. Mairal, et al. Sparse representation for computer vision and pattern recognition. Proceeding of the IEEE. 2010. 98: 1031-1044.

[12] L. Zhang, Y. Yang, X. Feng, Sparse Representation or Collaborative Representation: Which Helps Face Recognition, IEEE International Conference on Computer Vision, Barcelona, 2011,pp.471-481.

[13] D. M. W. Powers. Evaluation: From precision, recall and F-factor to ROC, informedness, markedness \& orrelation. J. Mach. Learn. Technol. 2011. 2: 37-63.

[14] Feng, H, Jiang, ZG, Xie, FY, et al. Automatic Fastener Classification and Defect Detection in Vision-Based Railway Inspection Systems, IEEE Transactions on Instrumentation and Measurement. 2014. 63: 877-888. 